\title{
Weighted Languages Recognizable by Weighted Tree Automata*
}

\author{
Zoltán Fülöp ${ }^{a}$ and Zsolt Gazdag ${ }^{a}$
}

\begin{abstract}
Yields of recognizable weighted tree languages, yields of local weighted tree languages, and weighted context-free languages are related. It is shown that the following five classes of weighted languages are the same: (i) the class of weighted languages generated by plain weighted context-free grammars, (ii) the class of weighted languages recognized by plain weighted tree automata, (iii) the class of weighted languages recognized by deterministic and plain topdown weighted tree automata, (iv) the class of weighted languages recognized by deterministic and plain bottom-up weighted tree automata, and (v) the class of weighted languages determined by plain weighted local systems.
\end{abstract}

\section{Introduction}

A tree automaton recognizes a set of trees over a ranked alphabet $\Sigma$ and a yield alphabet (or frontier alphabet) $X[14,15]$. Such trees are called $\Sigma X$-trees and the elements of $X$ may be leaves of $\Sigma X$-trees. Hence, a tree automaton also recognizes a language over $X$ as follows. For a $\Sigma X$-tree $\xi$, we define the yield yd $(\xi)$ of $\xi$ to be the string in $X^{*}$ obtained by reading the leaves of $\xi$ from left to right. Then, the language recognized by a tree automaton is the set of all strings $\operatorname{yd}(\xi)$, where $\xi$ is a tree recognized by the automaton.

The idea of using tree automata in the theory of languages was proposed already in papers [26], [20], [27] and [22]. Then, more results were obtained in [7], [23], [28], and [25], of which a summary can be found in $[14,15]$ (also, cf. $[10,6]$ ). Among other things, it was proved that the following four classes of languages are the same: (i) the class of context-free languages, (ii) the class of languages recognized by tree automata, (iii) the class of languages recognized by deterministic top-down tree automata, and (iv) the class of languages obtained by taking the yield of local tree languages (cf. Thm. II.9.4, III.2.7, and III. 2.9 in [14]).

With another line of research, tree automata were generalized to weighted tree automata (wta for short) $[2,1]$, in order to be able to deal with quantitative aspects

\footnotetext{
${ }^{*}$ This research was supported by the Hungarian Scientific Research Fund (OTKA) Grant K 108448.

${ }^{a}$ Department of Foundations of Computer Science, University of Szeged, Árpád tér 2, 6720 Szeged, Hungary, E-mail: \{fulop,gazdag\}@inf.u-szeged.hu
} 
of recognizable tree languages. A wta recognizes a weighted $\Sigma X$-tree language; that is, a mapping from the set of $\Sigma X$-trees to a weight structure. Here, we consider the case that the weight structure is a semiring $K$. For surveys, see [11, 13]; and note that in these papers weighted tree languages are called tree series. Also, weighted context-free languages were introduced under the name of algebraic power series [5]; see $[24,19]$ and $[21]$ for summary and [8] for a recent application ${ }^{1}$.

Weighted $\Sigma X$-tree languages with a yield alphabet and weighted languages over $X$ may be related as in the classical (unweighted) case. We can generalize the yield function to the weighted setting such that the yield $\operatorname{yd}(\Phi)$ of a weighted $\Sigma X$-tree language $\Phi$ will be a weighted language over $X$. In fact, the weight of a string $w \in X^{*}$ in $\operatorname{yd}(\Phi)$ is the sum of the weight of all trees in $\Phi$ of which the yield is $w$. We note that there may be infinitely many such trees, hence the sum may have infinitely many terms. In this case the semiring $K$ should be complete in the sense defined in [9].

The fundamental relation between recognizable weighted tree languages and weighted context-free languages is established in Thm. 8.6 and Cor. 8.7 of [11] in the form that, roughly speaking, algebraic power series are the same as yields of recognizable tree series. The authors use proof techniques, e.g. a theory of fixed points, which assume that the weight semiring is continuous (hence complete) and commutative. However, in some cases these strong assumptions are not necessary to achieve the same result. For instance, we do not need the assumption that $K$ is complete to define the weight of a string in a weighted context-free grammar if, for every $w \in X^{*}$, the set of derivation trees of $w$ with nonzero weight is finite (cf. the definition of the weighted CF grammar in [8]). The same holds for the yield of a weighted tree language $\Phi$ : we do not need the condition that $K$ is complete if, for every $w \in X^{*}$, the set of $\Sigma X$-trees $\xi$ with $\operatorname{yd}(\xi)=w$ and $\Phi(\xi) \neq 0$ is finite.

In this paper, we extend the above mentioned result of [11] to classes of weighted languages where the weight semiring is not commutative and not necessarily complete. Moreover, using the notions in [14], we will also take into consideration the weighted tree languages recognized by deterministic top-down wta and by deterministic bottom-up wta, as well as weighted languages obtained by taking the yield of local weighted tree languages [12]. For this, we adapt the definition of a weighted CF grammar of [8] to our semiring weighted context-free grammar and call this weighted context-free grammar plain. Moreover, we will introduce the concept of a plain wta and of a plain weighted local system, both as the counterpart of a plain weighted context-free grammar. Then, as the main result of the paper, we will show in Theorem 1 that the following five classes of weighted languages are the same: (i) the class of weighted languages generated by plain weighted context-free grammars, (ii) the class of weighted languages recognized by plain wta, (iii) the class of weighted languages recognized by deterministic and plain top-down wta, (iv) the class of weighted languages recognized by deterministic and plain bottomup wta, and (v) the class of weighted languages determined by plain weighted local systems.

\footnotetext{
${ }^{1}$ The weight structure in $[8]$ is a valuation monoid, which is a generalization of a semiring.
} 


\section{Preliminaries}

\subsection{General concepts}

First, let $\mathbb{N}$ be the set of positive integers and $\mathbb{N}_{0}$ be the set of nonnegative integers. For every $k \in \mathbb{N}$, we define $[k]=\{1, \ldots, k\}$.

An alphabet is a finite set $X$ of symbols. We denote by $X^{*}$ the set of all words (or strings) over $X$ and by $\varepsilon$ the empty string. The length of a string $w \in X^{*}$ is denoted by $|w|$. A language $L$ (over $X$ ) is an arbitrary subset of $X^{*}$.

A ranked alphabet is a tuple $(\Sigma, r k)$ where $\Sigma$ is an alphabet and rk: $\Sigma \rightarrow \mathbb{N}_{0}$ is the rank mapping. For every $k \geq 0$, we define $\Sigma_{k}=\{\sigma \in \Sigma \mid \operatorname{rk}(\sigma)=k\}$. Sometimes we write $\sigma^{(k)}$ to mean that $\sigma \in \Sigma_{k}$. Moreover, let $X$ be a set disjoint with $\Sigma$. The set of terms (or: trees) over $X$, denoted by $T_{\Sigma}(X)$, is the smallest set $T$ such that (i) $\Sigma_{0} \cup X \subseteq T$ and (ii) if $k \geq 1, \sigma \in \Sigma_{k}$, and $\xi_{1}, \ldots, \xi_{k} \in T$, then $\sigma\left(\xi_{1}, \ldots, \xi_{k}\right) \in T$. We shall abbreviate $T_{\Sigma}(\emptyset)$ by $T_{\Sigma}$.

We define the mapping pos : $T_{\Sigma}(X) \rightarrow \mathcal{P}\left(\mathbb{N}^{*}\right)$ by recursion as follows: (i) for each $y \in\left(\Sigma_{0} \cup X\right)$ we let $\operatorname{pos}(y)=\{\varepsilon\}$ and (ii) for every $k \geq 1, \sigma \in \Sigma^{(k)}$, and $\xi_{1}, \ldots, \xi_{k} \in T_{\Sigma}(X)$ we let $\operatorname{pos}\left(\sigma\left(\xi_{1}, \ldots, \xi_{k}\right)\right)=\{\varepsilon\} \cup\left\{i p \mid i \in[k], p \in \operatorname{pos}\left(\xi_{i}\right)\right\}$. For every $\xi \in T_{\Sigma}(X)$ we call $\operatorname{pos}(\xi)$ the set of positions in $\xi$ and, for every $p \in \operatorname{pos}(\xi)$, we define the label $\xi(p) \in \Sigma$ of $\xi$ at position $p$ and the subtree $\left.\xi\right|_{p} \in T_{\Sigma}(X)$ of $\xi$ at position $p$ in the usual way (cf. e.g. [13]). We shall call $\xi(\varepsilon)$ the root of $\xi$ and denote it by $\operatorname{rt}(\xi)$.

A monoid $(K,+, 0)$ is commutative if $a+b=b+a$ and zero-sum free if $a+b=0$ implies $a=b=0$ for every $a, b \in K$. We extend the binary summation + to a sum operation $\sum_{I}: K^{I} \rightarrow K$ for each finite index set $I$ in the usual way. For each finite family $\left(a_{i} \mid i \in I\right)$ of elements of $K$ we write the sum $\sum_{I}\left(a_{i} \mid i \in I\right)$ also in the form $\sum\left(a_{i} \mid i \in I\right)$ or $\sum_{i \in I} a_{i}$. Moreover, the monoid $(K,+, 0)$ is complete if it has a sum operation $\sum_{I}: K^{I} \rightarrow K$ for each countable index set $I$ such that this sum coincides with the extension of + when $I$ is finite (for the axioms, see [9, p. 124]). For countable index sets $I$ and families $\left(a_{i} \mid i \in I\right)$ we will also use the notation $\sum\left(a_{i} \mid i \in I\right)$ and $\sum_{i \in I} a_{i}$ in the same sense as that for finite index sets and families.

A semiring is an algebra $(K,+, \cdot, 0,1)$ which consists of a commutative monoid $(K,+, 0)$, called the additive monoid, and a monoid $(K, \cdot 1)$, called the multiplicative monoid of the semiring, such that multiplication distributes (from both left and right) over addition, and moreover, $0 \neq 1$ and 0 is absorbing with respect to . (also both from left and right). We call the semiring zero-sum free if its additive monoid is zero-sum free and commutative if its multiplicative monoid is commutative. Furthermore, the semiring is complete if its additive monoid is complete and the generalized distributivity law holds for infinite sums (see [9, p. 124]). An introduction to and some details about semirings can be found e.g. in [17, 18]. As usual, we often denote a semiring by its carrier set.

In the rest of this paper $\Sigma$ will denote an arbitrary ranked alphabet, $X$ will denote an arbitrary alphabet which is disjoint with $\Sigma$, and $K$ will denote an arbitrary semiring, unless specified otherwise. 
A $K$-weighted tree language is a mapping $\Phi: T_{\Sigma}(X) \rightarrow K$. For every $\xi \in T_{\Sigma}(X)$, the element $\Phi(\xi)$ of $K$ is called the weight of $\xi$ (in $\Phi$ ). Analogously, a $K$-weighted language is a mapping $\lambda: X^{*} \rightarrow K$ and, for every $w \in X^{*}$, the element $\lambda(w)$ of $K$ is called the weight of $w$ (in $\lambda$ ). Sometimes we drop $K$ from $K$-weighted and thus we speak about a weighted (tree) language.

Next, we define the yield of weighted tree languages which satisfies a certain condition. For this, first we define the yield of a tree in $T_{\Sigma}(X)$ by the function $\operatorname{yd}_{\Sigma}: T_{\Sigma}(X) \rightarrow X^{*}$ as follows: (i) for every $y \in\left(\Sigma_{0} \cup X\right)$ let $\operatorname{yd}_{\Sigma}(y)=\varepsilon$ if $y \in \Sigma_{0}$ and $\operatorname{yd}_{\Sigma}(y)=y$ if $y \in X$, and (ii) for every $\xi=\sigma\left(\xi_{1}, \ldots, \xi_{k}\right)$, where $k \geq 1$, we define $\operatorname{yd}_{\Sigma}(\xi)=\operatorname{yd}_{\Sigma}\left(\xi_{1}\right) \ldots \operatorname{yd}_{\Sigma}\left(\xi_{k}\right)$. Hence, we have $\operatorname{yd}_{\Sigma}^{-1}(w)=\left\{\xi \in T_{\Sigma}(X) \mid\right.$ $\left.\operatorname{yd}_{\Sigma}(\xi)=w\right\}$ for every $w \in X^{*}$.

Now let $\Phi: T_{\Sigma}(X) \rightarrow K$ be a weighted tree language. We call $\Phi$ summable for yield (or: summable) if the semiring $K$ is complete or the set

$$
T_{\Phi}(w)=\left\{\xi \in \operatorname{yd}_{\Sigma}^{-1}(w) \mid \Phi(\xi) \neq 0\right\}
$$

is finite for every $w \in X^{*}$. If $\Phi$ is summable, then we define the yield of $\Phi$ to be the weighted language $\mathrm{yd}(\Phi): X^{*} \rightarrow K$ by

$$
\operatorname{yd}(\Phi)(w)=\sum_{\xi \in T_{\Phi}(w)} \Phi(\xi)
$$

for every $w \in X^{*}$, where $\sum$ denotes the extension of the addition of $K$. (The fact that $\Phi$ is summable guarantees that the above sum is well-defined.) Moreover, for a class $C(K)$ of summable $K$-weighted languages we define $\mathrm{yd}^{\prime}(C(K))=\{\mathrm{yd}(\Phi) \mid$ $\Phi \in C(K)\}$ and we will write yd for $\mathrm{yd}^{\prime}$ in the rest of the paper.

\subsection{Weighted context-free languages}

Weighted context-free grammars over semirings were introduced in [5] (see also $[24,19])$. Recently, a Chomsky-Schützenberger theorem was proved for weighted context-free grammars over tree valuation monoids in [8]. We follow the idea of [8] to define the semantics of a weighted context-free grammar, but we will use semirings as weight structures.

A $K$-weighted context-free grammar (or $\mathrm{CF}(K)$-grammar for short) is a tuple $\mathcal{G}=(N, X, Z, P, \mathrm{wt})$, where $N$ and $X$ are alphabets (nonterminals and terminals, respectively) such that $N \cap X=\emptyset, Z \in N$ (initial nonterminal), $P$ is a finite set of rules of the form $A \rightarrow \alpha$, where $A \in N$ and $\alpha \in(N \cup X)^{*}$, and wt: $P \rightarrow K$ is a mapping (weight assignment). Given a rule $r=(A \rightarrow \alpha)$, we call the nonterminal $A$ the left-hand side of $r$ and denote it by $\operatorname{lhs}(r)$.

The semantics of a weighted context-free grammar is defined in [8] in terms of leftmost derivations. Here, we follow an equivalent approach and use derivation trees in the sense of $[16$, Sect. 3.1]. In fact, we will treat $P$ as a ranked alphabet by letting $\operatorname{rk}(r)=|\alpha|$ for every $r=(A \rightarrow \alpha) \in P$ and we will denote this ranked alphabet by $\bar{P}$. Hence $\bar{P}_{k}=\{(A \rightarrow \alpha) \in P|| \alpha \mid=k\}$ for every $k \geq 0$.

We can extend the mapping wt to trees in $T_{\bar{P}}(X)$ by defining the mapping wtt $^{\prime}: T_{\bar{P}}(X) \rightarrow K$ as follows. For every $\zeta \in T_{\bar{P}}(X)$, 
(i) if $\zeta=r$ for some rule $r \in \bar{P}_{0}$, then $\mathrm{wt}^{\prime}(\zeta)=\mathrm{wt}(r)$,

(ii) if $\zeta \in X$, then $\operatorname{wt}^{\prime}(\zeta)=1$, and

(iii) if $\zeta=r\left(\zeta_{1}, \ldots, \zeta_{k}\right)$, for some $k \geq 1, r \in \bar{P}_{k}$, and $\zeta_{1}, \ldots, \zeta_{k} \in T_{\bar{P}}(X)$, then $\mathrm{wt}^{\prime}(\zeta)=\mathrm{wt}^{\prime}\left(\zeta_{1}\right) \cdot \ldots \cdot \mathrm{wt}^{\prime}\left(\zeta_{k}\right) \cdot \mathrm{wt}(r)($ where $\cdot$ is the multiplication of $K)$.

We note that $\mathrm{wt}^{\prime}$ is a $K$-weighted tree language, thus we may call $\mathrm{wt}^{\prime}(\zeta)$ the weight of $\zeta$. From now on, we write wt for $\mathrm{wt}^{\prime}$.

Next, we define derivation trees as certain trees in $T_{\bar{P}}(X)$. Formally, for every $w \in X^{*}$, we define the set $D_{\mathcal{G}}(w)$ of derivation trees of $w$ such that, for every $\zeta \in T_{\bar{P}}(X)$, we have $\zeta \in D_{\mathcal{G}}(w)$, if and only if

- $\operatorname{lhs}(\operatorname{rt}(\zeta))=Z$ and $\operatorname{yd}_{\bar{P}}(\zeta)=w$,

- for every $p \in \operatorname{pos}(\zeta)$ with $\zeta(p)=\left(A \rightarrow \alpha_{1} \ldots \alpha_{k}\right)$ for some $k \geq 1$ and $\alpha_{1}, \ldots, \alpha_{k} \in(N \cup X)$, we have $\zeta(p i)=y_{i}$, where

$$
y_{i}= \begin{cases}\alpha_{i} & \text { if } \alpha_{i} \in X \\ \text { a rule } r_{i} \in \bar{P} \text { with } \operatorname{lhs}\left(r_{i}\right)=\alpha_{i} & \text { if } \alpha_{i} \in N,\end{cases}
$$

for every $1 \leq i \leq k$.

The following concept was suggested by [8]. However, we will use a new name to identify the defined class of weighted context-free grammars. We call $\mathcal{G}$ plain if the semiring $K$ is complete or the set $\left\{\zeta \in D_{\mathcal{G}}(w) \mid \operatorname{wt}(\zeta) \neq 0\right\}$ is finite for every $w \in X^{*}$. In this case we define the weighted language generated by $\mathcal{G}$ to be the $K$-weighted language $\lambda_{\mathcal{G}}: X^{*} \rightarrow K$ given for every $w \in X^{*}$ by

$$
\lambda_{\mathcal{G}}(w)=\sum_{\zeta \in D_{\mathcal{G}}(w), \operatorname{wt}(\zeta) \neq 0} \mathrm{wt}(\zeta) .
$$

The class of weighted languages generated by plain $\mathrm{CF}(K)$-grammars is denoted by $\operatorname{CFL}_{\mathrm{p}}(K)$.

Example 1. It is known that the language $L=\left\{\left.w \in\{0,1\}^{*}|| w\right|_{0}=|w|_{1}\right\}$ is context-free. It can be generated, for instance, by the context-free grammar

$$
r_{1}: S \rightarrow S S, r_{2}: S \rightarrow 0 S 1, r_{3}: S \rightarrow 1 S 0, \text { and } r_{4}: S \rightarrow \varepsilon .
$$

This grammar is ambiguous; that is, there are words in $L$ which have more than one derivation tree.

Now we will consider the tropical semiring Trop $=(\mathbb{N} \cup\{\infty\}$, min, $+, \infty, 0)$. It is well known that Trop is complete. Then we define the $\mathrm{CF}$ (Trop)-grammar $\mathcal{G}=(\{S\}, X, S, P, \mathrm{wt})$, where $X=\{0,1\}, P=\left\{r_{1}, r_{2}, r_{3}, r_{4}\right\}, \operatorname{wt}\left(r_{1}\right)=\operatorname{wt}\left(r_{2}\right)=$ $\operatorname{wt}\left(r_{3}\right)=0$, and $\operatorname{wt}\left(r_{4}\right)=1$. The grammar $\mathcal{G}$ is plain, because Trop is complete. In Figure 1, we show two trees in $T_{\bar{P}}(X)$, where the rank of $r_{1}, r_{2}, r_{3}$, and $r_{4}$ in $\bar{P}$ is $2,3,3$, and 0 , respectively. The first tree (from left to right) is not a derivation 

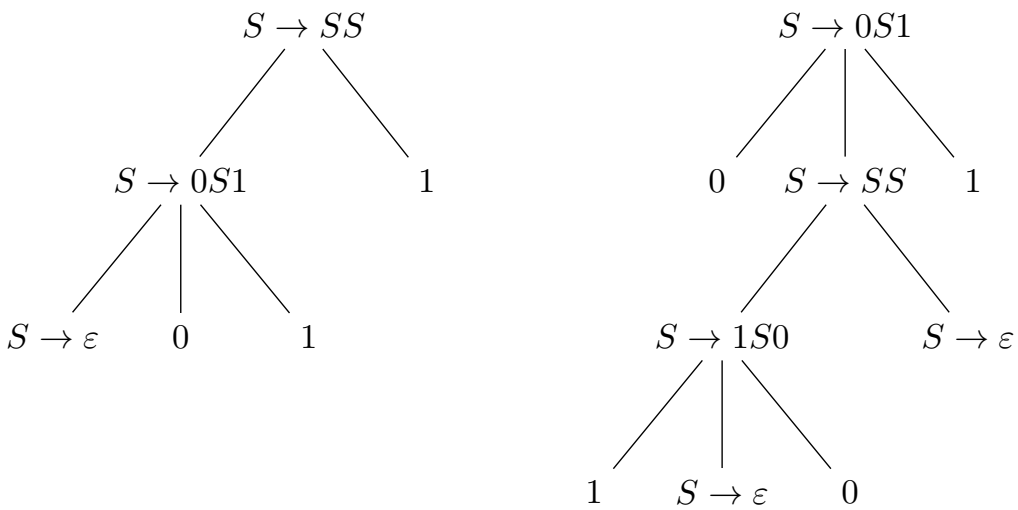

Figure 1: Two trees in $T_{\bar{P}}(X)$ of Example 1.

tree of any $w \in X^{*}$, while the second one is a derivation tree of 0101 , i.e. it is in $D_{\mathcal{G}}(0101)$. The weight of the first tree is 1 and the weight of the second one is 2 .

Now let $w \in \Sigma^{*}$. It is clear that for every $\zeta \in T_{\bar{P}}(X)$, the weight of $\zeta$ is the number of the occurrences of $r_{4}$ (roughly speaking, the number of erasing rules) in $\zeta$. Let us denote this number by $\#_{\text {ers }}(\zeta)$. Moreover,

$$
\lambda_{\mathcal{G}}(w)=\min \left(\operatorname{wt}(\zeta) \mid \zeta \in D_{\mathcal{G}}(w), \operatorname{wt}(\zeta) \neq \infty\right)=\min \left(\# \text { ers }(\zeta) \mid \zeta \in D_{\mathcal{G}}(w)\right)
$$

\subsection{Recognizable weighted tree languages}

A $K$-weighted tree automaton with yield alphabet (or $K$-wta for short) is a tuple $\mathcal{A}=(Q, \Sigma, X, \delta, \kappa)$ where $Q$ is a finite nonempty set, the set of states, $\Sigma$ is the ranked input alphabet, $X$ is the yield alphabet, $\delta=\left(\delta_{k} \mid k \in \mathbb{N}_{0}\right)$ is a family of transition mappings ${ }^{2}$ such that

$$
\delta_{k}: Q^{k} \times \Sigma_{k} \times Q \rightarrow K \text { for } k \geq 1 \text { and } \delta_{0}:\left(\Sigma_{0} \cup X\right) \times Q \rightarrow K
$$

and $\kappa: Q \rightarrow K$ is the root weight mapping.

For every $k \in \mathbb{N}$ we call an element $\left(q_{1} \ldots q_{k}, \sigma, q\right) \in Q^{k} \times \Sigma_{k} \times Q$ a transition, and call $\delta_{k}\left(q_{1} \ldots q_{k}, \sigma, q\right) \in K$ the weight of that transition. (Here and in the rest of the paper, we abbreviate $\left(q_{1}, \ldots, q_{k}\right)$ by $q_{1} \ldots q_{k}$.)

Let $\xi \in T_{\Sigma}(X)$. A run of $\mathcal{A}$ on $\xi$ is a mapping $\omega: \operatorname{pos}(\xi) \rightarrow Q$. The set of all runs of $\mathcal{A}$ on $\xi$ is denoted by $R_{\mathcal{A}}(\xi)$. For every $\omega \in R_{\mathcal{A}}(\xi)$ and $p \in \operatorname{pos}(\xi)$, the run $\left.\omega\right|_{p}$ of $\mathcal{A}$ on $\left.\xi\right|_{p}$ is defined by $\left.\omega\right|_{p}\left(p^{\prime}\right)=\omega\left(p p^{\prime}\right)$ for every $p^{\prime} \in \operatorname{pos}\left(\left.\xi\right|_{p}\right)$. Now we define the weight of a run $\omega \in R_{\mathcal{A}}(\xi)$ to be an element $\delta^{*}(\omega)$ of $K$ by induction as follows:

\footnotetext{
${ }^{2}$ In the literature $\delta$ is also called a tree representation and $\delta_{k}$ is given as a mapping of type $\Sigma_{k} \rightarrow S^{Q^{k} \times Q}$
} 
- if $\xi=y \in\left(\Sigma_{0} \cup X\right)$, then $\delta^{*}(\omega)=\delta_{0}(y, \omega(\varepsilon))$,

- if $\xi=\sigma\left(\xi_{1}, \ldots, \xi_{k}\right)$ for some $k \geq 1$, then

$$
\delta^{*}(\omega)=\delta^{*}\left(\left.\omega\right|_{1}\right) \cdot \ldots \cdot \delta^{*}\left(\left.\omega\right|_{k}\right) \cdot \delta_{k}(\omega(1) \ldots \omega(k), \sigma, \omega(\varepsilon)),
$$

where $\cdot$ is the product of the semiring $K$. (Note that $\left.\omega\right|_{i} \in R_{\mathcal{A}}\left(\xi_{i}\right)$ because $\xi_{i}=\left.\xi\right|_{i}$ for every $\left.1 \leq i \leq k\right)$.

The $K$-weighted tree language $\|\mathcal{A}\|: T_{\Sigma}(X) \rightarrow K$ recognized by $\mathcal{A}$ is defined by

$$
\|\mathcal{A}\|(\xi)=\sum_{\omega \in R_{\mathcal{A}}(\xi)} \delta^{*}(\omega) \cdot \kappa(\omega(\varepsilon))
$$

for every $\xi \in T_{\Sigma}(X)$. An introduction to the theory of wta over semirings and some results can be found in [4], [11], and [13].

Example 2. (Cf. [3, Example 3.3]) We consider the arctic semiring Arct $=(\mathbb{N} \cup$ $\{-\infty\}, \max ,+,-\infty, 0)$ and construct the wta $\mathcal{A}=(Q, \Sigma, X, \delta, \kappa)$ which recognizes the weighted tree language height $: T_{\Sigma}(X) \rightarrow \mathbb{N}$, where height $(\xi)=\max \{|w| \mid w \in$ $\operatorname{pos}(\xi)\}$. For this, let $Q=\left\{p_{1}, p_{2}\right\}, \Sigma=\left\{\sigma^{(2)}, \alpha^{(0)}\right\}, X=\left\{x_{1}, x_{2}\right\}$. Furthermore, let

$$
\begin{array}{ll}
\delta_{0}\left(y, p_{1}\right) & =\delta_{0}\left(y, p_{2}\right) \\
\delta_{2}\left(p_{1} p_{2}, \sigma, p_{1}\right) & =\delta_{2}\left(p_{2} p_{1}, \sigma, p_{1}\right)=1, \text { for all } y \in\left(\Sigma_{0} \cup X\right), \\
\delta_{2}\left(p_{2} p_{2}, \sigma, p_{2}\right) & =0,
\end{array}
$$

and for every other transition $\left(q_{1} q_{2}, \sigma, q\right)$ we have $\delta_{2}\left(q_{1} q_{2}, \sigma, q\right)=-\infty$. Lastly, let $\kappa\left(p_{1}\right)=0$ and $\kappa\left(p_{2}\right)=-\infty$.

Intuitively, $\mathcal{A}$ works as follows. For every input tree $\xi$ and run $\omega \in R_{\mathcal{A}}(\xi)$,

- if $\omega$ assigns $p_{1}$ to each position in a path from the root to a leaf of $\xi$ (in particular, to the root and to that leaf of $\xi$ ) and assigns $p_{2}$ to every other position in $\xi$, then the weight of $\omega$ is equal to the length of that path,

- if $\omega$ assigns $p_{2}$ to each position in $\xi$, then the weight of $\omega$ is 0 , and

- in every other case, the weight of $\omega$ is $-\infty$.

Hence,

$$
\begin{aligned}
& \max \left(\delta^{*}(\omega) \mid \omega \in R_{\mathcal{A}}(\xi), \omega(\varepsilon)=p_{1}\right)=\operatorname{height}(\xi) \text { and } \\
& \max \left(\delta^{*}(\omega) \mid \omega \in R_{\mathcal{A}}(\xi), \omega(\varepsilon)=p_{2}\right)=0,
\end{aligned}
$$

for every $\xi \in T_{\Sigma}(X)$. Thus,

$$
\begin{aligned}
& \|\mathcal{A}\|(\xi)=\max \left(\delta^{*}(\omega)+\kappa(\omega(\varepsilon)) \mid \omega \in R_{\mathcal{A}}(\xi)\right)= \\
& \max \left(\max \left(\delta^{*}(\omega)+\kappa\left(p_{1}\right) \mid \omega \in R_{\mathcal{A}}(\xi), \omega(\varepsilon)=p_{1}\right),\right. \\
& \left.\max \left(\delta^{*}(\omega)+\kappa\left(p_{2}\right) \mid \omega \in R_{\mathcal{A}}(\xi), \omega(\varepsilon)=p_{2}\right)\right)= \\
& \max (\operatorname{height}(\xi)+0,0+(-\infty))=\operatorname{height}(\xi) .
\end{aligned}
$$


A $K$-wta $\mathcal{A}=(Q, \Sigma, X, \delta, \kappa)$ is bottom-up deterministic (or bu-deterministic) if for every $y \in\left(\Sigma_{0} \cup X\right)$, there is at most one $q \in Q$ such that $\delta_{0}(y, q) \neq 0$, and for every $k \geq 1, \sigma \in \Sigma_{k}$, and $w \in Q^{k}$ there is at most one $q \in Q$ such that $\delta_{k}(w, \sigma, q) \neq 0$. If this is the case, then for every input tree $\xi \in T_{\Sigma}(X)$, there is at most one $\omega \in R_{\mathcal{A}}(\xi)$ such that $\delta^{*}(\omega) \neq 0$. Thus $\|\mathcal{A}\|(\xi)=\delta^{*}(\omega) \cdot \kappa(\omega(\varepsilon))$, if $\omega$ is the only element of $R_{\mathcal{A}}(\xi)$ with $\delta^{*}(\omega) \neq 0$ and $\|\mathcal{A}\|(\xi)=0$ if there is no such element in $R_{\mathcal{A}}(\xi)$.

Moreover, $\mathcal{A}$ is top-down deterministic (or td-deterministic) if the set $\{q \in Q \mid$ $\kappa(q) \neq 0\}$ is a singleton, for every $y \in\left(\Sigma_{0} \cup X\right)$, there is at most one $q \in Q$ such that $\delta_{0}(y, q) \neq 0$, and for every $k \geq 1, \sigma \in \Sigma_{k}$, and $q \in Q$ there is at most one $w \in Q^{k}$ such that $\delta_{k}(w, \sigma, q) \neq 0$. In this case, for every $q \in Q$ and $\xi \in T_{\Sigma}(X)$, there is at most one $\omega \in R_{\mathcal{A}}(\xi)$ with $\omega(\varepsilon)=q$ and $\delta^{*}(\omega) \neq 0$. Hence the formula for $\|\mathcal{A}\|(\xi)$ can be simplified in the same way as for a bu-deterministic $K$-wta. Let us mention that for both kinds of deterministic $K$-wta, the addition + of $K$ is not used to the compute $\|\mathcal{A}\|$.

A $K$-weighted tree language $\Phi: T_{\Sigma}(X) \rightarrow K$ is recognizable (budeterministically recognizable, td-deterministically recognizable) if there is a $K$-wta (resp. bu-deterministic $K$-wta, td-deterministic $K$-wta) $\mathcal{A}$ such that $\Phi=\|\mathcal{A}\|$. The class of all summable and recognizable $K$-weighted tree languages is denoted

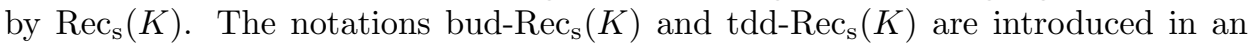
analogous way.

\subsection{Local weighted tree languages}

Local weighted tree languages were introduced in [12]. Here, we give a slightly more general definition by using a yield alphabet $X$ in order to be able to handle yields of local weighted tree languages.

We introduce the family $\operatorname{Fork}(\Sigma, X)=\left(\operatorname{Fork}_{k}(\Sigma, X) \mid k \geq 0\right)$ of sets, where

$$
\operatorname{Fork}_{k}(\Sigma, X)=(\Sigma \cup X)^{k} \times \Sigma_{k} \text { for } k \geq 1 \text { and } \operatorname{Fork}_{0}(\Sigma, X)=\Sigma_{0} \cup X \text {. }
$$

We write the elements of $\operatorname{Fork}_{k}(\Sigma, X), k \geq 1$ in the form $\left(y_{1} \ldots y_{k}, \sigma\right)$ and call them $(\Sigma, X)$-forks. A fork $\left(y_{1} \ldots y_{k}, \sigma\right)$ occurs in a tree if the tree has a $\sigma$-node of which the $k$ children are labeled by $y_{1}, \ldots, y_{k}$ from the left to right.

A $K$-weighted local system (or $K$-wls for short) is a system $\mathcal{L}=(\Sigma, X, \varphi, \rho)$, where $\varphi$ is a family of mappings $\left(\varphi_{k} \mid k \geq 0\right)$ with

$$
\varphi_{k}: \operatorname{Fork}_{k}(\Sigma \cup X) \rightarrow K, \text { and } \rho:(\Sigma \cup X) \rightarrow K
$$

is another mapping. Intuitively, we associate a weight, i.e., an element of $K$ with each fork and also with each symbol in $\Sigma \cup X$. Note that this weight may be 0 .

Next, we define the $K$-weighted tree language determined by $\mathcal{L}$. For this, we extend $\varphi$ to the mapping $\varphi^{\prime}: T_{\Sigma}(X) \rightarrow K$ defined by induction as follows:

(i) $\varphi^{\prime}(y)=\varphi_{0}(y)$ for every $y \in\left(\Sigma_{0} \cup X\right)$,

(ii) $\varphi^{\prime}\left(\sigma\left(\xi_{1}, \ldots, \xi_{k}\right)\right)=\varphi^{\prime}\left(\xi_{1}\right) \cdot \ldots \cdot \varphi^{\prime}\left(\xi_{k}\right) \cdot \varphi_{k}\left(\operatorname{rt}\left(\xi_{1}\right) \ldots \operatorname{rt}\left(\xi_{k}\right), \sigma\right)$ for every $k \geq 1$, $\sigma \in \Sigma_{k}$, and $\xi_{1}, \ldots, \xi_{k} \in T_{\Sigma}(X)$. 
In the following we write $\varphi$ for $\varphi^{\prime}$. The $K$-weighted tree language $\|\mathcal{L}\|: T_{\Sigma}(X) \rightarrow K$ determined by $\mathcal{L}$ is defined by $\|\mathcal{L}\|(\xi)=\varphi(\xi) \cdot \rho(\operatorname{rt}(\xi))$ for every $\xi \in T_{\Sigma}(X)$. As for deterministic $K$-wta, the operation + of $K$ is not used in the definition of $\|\mathcal{L}\|$.

Thus, $\varphi(\xi)$ is the (semiring) product of the weights associated with the forks in $\xi$. The order of the factors is the postorder of the nodes of $\xi$. Also, the weight $\|\mathcal{L}\|(\xi)$ of $\xi$ is the product of $\varphi(\xi)$ and the weight associated to the root of $\xi$.

Example 3. We consider again the ranked alphabet $\Sigma=\left\{\sigma^{(2)}, \alpha^{(0)}\right\}$, the set $X=\left\{x_{1}, x_{2}\right\}$ and the semiring Arct. We define the Arct-wls $\mathcal{L}=(\Sigma, X, \varphi, \rho)$ by

- $\varphi_{2}(y \alpha, \sigma)=1, \varphi_{2}(y z, \sigma)=0$ for all $y, z \in(\Sigma \cup X)$ with $z \neq \alpha$, and $\varphi_{0}(y)=0$ for all $y \in\left(\Sigma_{0} \cup X\right)$, and

- $\rho(y)=0$ for all $y \in(\Sigma \cup X)$.

It should be clear that $\|\mathcal{L}\|(\xi)$ is the number of the occurrences of the pattern $\sigma\left({ }_{-}, \alpha\right)$ in $\xi$ for every $\xi \in T_{\Sigma}(X)$, where ', ' is a placeholder which may be filled by any element of $\Sigma \cup X$. We note that in [13, Example 3.4] a wta is given over the semiring of natural numbers which recognizes $\|\mathcal{L}\|$ (with the difference being that there $X=\emptyset)$.

A $K$-weighted tree language $\Phi: T_{\Sigma}(X) \rightarrow K$ is called local if there is a $K$-wls $\mathcal{L}$ such that $\Phi=\|\mathcal{L}\|$.

\section{The results}

Now, we will introduce plain wta and plain wls and define weighted languages recognizable by plain wta and determined by plain wls, respectively. We relate the class of weighted languages generated by plain weighted context-free grammars, the class of weighted languages recognizable by plain wta, and the class of weighted languages determined by plain wls.

We say that a $K$-wta $\mathcal{A}=(Q, \Sigma, X, \delta, \kappa)$ is plain if $K$ is complete or, for every $w \in \Sigma^{*}$, the set

$$
U_{\mathcal{A}}(w)=\left\{\xi \in \operatorname{yd}_{\Sigma}^{-1}(w) \mid \exists\left(\omega \in R_{\mathcal{A}}(\xi)\right): \delta^{*}(\omega) \cdot \kappa(\omega(\varepsilon)) \neq 0\right\}
$$

is finite.

Lemma 1. Let $\mathcal{A}=(Q, \Sigma, X, \delta, \kappa)$ be a $K$-wta.

(1) If $\mathcal{A}$ is plain, then $\|\mathcal{A}\|$ is summable and

$$
\operatorname{yd}(\|\mathcal{A}\|)(w)=\sum_{\xi \in U_{\mathcal{A}}(w), \omega \in R_{\mathcal{A}}(\xi)} \delta^{*}(\omega) \cdot \kappa(\omega(\varepsilon))
$$

for every $w \in \Sigma^{*}$. 
(2) If $K$ is zero-sum free and $\|\mathcal{A}\|$ is summable, then $\mathcal{A}$ is plain.

(3) If $\mathcal{A}$ is bu-deterministic and $\|\mathcal{A}\|$ is summable, then $\mathcal{A}$ is plain. The same holds when we replace bu-deterministic by td-deterministic.

Proof. Let $w \in \Sigma^{*}$. It is obvious that

$$
\begin{aligned}
& T_{\|\mathcal{A}\|}(w)= \\
& \left\{\xi \in \operatorname{yd}_{\Sigma}^{-1}(w) \mid\|\mathcal{A}\|(\xi) \neq 0\right\}=\left\{\xi \in \operatorname{yd}_{\Sigma}^{-1}(w) \mid\left(\sum_{\omega \in R_{\mathcal{A}}(\xi)} \delta^{*}(\omega) \cdot \kappa(\omega(\varepsilon))\right) \neq 0\right\} \subseteq \\
& \left\{\xi \in \operatorname{yd}_{\Sigma}^{-1}(w) \mid \exists\left(\omega \in R_{\mathcal{A}}(\xi)\right): \delta^{*}(\omega) \cdot \kappa(\omega(\varepsilon)) \neq 0\right\}=U_{\mathcal{A}}(w) .
\end{aligned}
$$

Now, we will prove (1). Since $\mathcal{A}$ is plain, the set $U_{\mathcal{A}}(w)$ is finite. Thus $T_{\|\mathcal{A}\|}(w)$ is also finite, hence $\|\mathcal{A}\|$ is summable. Moreover,

$$
\begin{gathered}
\operatorname{yd}(\|\mathcal{A}\|)(w)=\sum_{\xi \in T_{\|\mathcal{A}\|}(w)}\|\mathcal{A}\|(\xi)=\sum_{\xi \in T_{\|\mathcal{A}\|}(w)}\left(\sum_{\omega \in R_{\mathcal{A}}(\xi)} \delta^{*}(\omega) \cdot \kappa(\omega(\varepsilon))\right)= \\
\sum_{\xi \in U_{\mathcal{A}}(w)}\left(\sum_{\omega \in R_{\mathcal{A}}(\xi)} \delta^{*}(\omega) \cdot \kappa(\omega(\varepsilon))\right)=\sum_{\xi \in U_{\mathcal{A}}(w), \omega \in R_{\mathcal{A}}(\xi)} \delta^{*}(\omega) \cdot \kappa(\omega(\varepsilon)),
\end{gathered}
$$

where the third equality holds because for every $\xi \in\left(U_{\mathcal{A}}(w) \backslash T_{\|\mathcal{A}\|}(w)\right)$ the corresponding sum is 0 and the fourth one holds because summation is associative and commutative in $K$.

To prove (2), we assume that $K$ is zero-sum free and that $\|\mathcal{A}\|$ is summable. Due to the fact that $K$ is zero-sum free, $\subseteq$ becomes an equality and therefore $T_{\|\mathcal{A}\|}(w)=U_{\mathcal{A}}(w)$. Since $T_{\|\mathcal{A}\|}(w)$ is finite, the set $U_{\mathcal{A}}(w)$ is also finite and hence $\mathcal{A}$ is plain.

Statement (3) follows from the fact that, by the remarks we made on the runs of bu-deterministic wta and td-deterministic wta, $\subseteq$ becomes an equality and so we have again that $T_{\|\mathcal{A}\|}(w)=U_{\mathcal{A}}(w)$.

Let $\operatorname{Rec}_{\mathrm{p}}(K)$ be the class of all $K$-weighted tree languages which are recognizable by a plain $K$-wta. The notations bud- $\operatorname{Rec}_{\mathrm{p}}(K)$ and tdd- $\operatorname{Rec}_{\mathrm{p}}(K)$ are introduced in an analogous way.

Let $\mathcal{A}$ be a plain $K$-wta. Then we call $\mathrm{yd}(\|\mathcal{A}\|)$ the weighted language recognized by $\mathcal{A}$ and denote it by $\lambda_{\mathcal{A}}$. Note that

$$
\lambda_{\mathcal{A}}=\sum_{\xi \in U_{\mathcal{A}}(w), \omega \in R_{\mathcal{A}}(\xi)} \delta^{*}(\omega) \cdot \kappa(\omega(\varepsilon)) .
$$

The next statements immediately follow from Lemma 1.

Corollary 1. (1) $\mathrm{yd}\left(\operatorname{Rec}_{\mathrm{p}}(K)\right) \subseteq \mathrm{yd}\left(\operatorname{Rec}_{\mathrm{s}}(K)\right)$. 
(2) If $K$ is zero-sum free, then $\operatorname{yd}\left(\operatorname{Rec}_{\mathrm{p}}(K)\right)=\operatorname{yd}\left(\operatorname{Rec}_{\mathrm{s}}(K)\right)$.

(3) $\operatorname{yd}\left(\operatorname{bud}_{-} \operatorname{Rec}_{\mathrm{p}}(K)\right)=\operatorname{yd}\left(\operatorname{bud}_{-}-\operatorname{Rec}_{\mathrm{s}}(K)\right)$.

(4) $\operatorname{yd}\left(\operatorname{tdd}-\operatorname{Rec}_{\mathrm{p}}(K)\right)=\mathrm{yd}\left(\operatorname{tdd}-\operatorname{Rec}_{\mathrm{s}}(K)\right)$.

It is an open question whether there is a semiring $K$ such that $\operatorname{yd}\left(\operatorname{Rec}_{\mathrm{s}}(K)\right) \backslash$ $\operatorname{yd}\left(\operatorname{Rec}_{\mathrm{p}}(K)\right) \neq \emptyset$. However, we can prove the following weaker statement.

Lemma 2. There is a semiring $K$ and a $K$-wta $\mathcal{A}$ which is not plain such that $\|\mathcal{A}\|$ is summable.

Proof. We consider the semiring $(\mathbb{Z},+, \cdot, 0,1)$ of integers. We note that $\mathbb{Z}$ is not zero-sum free. Moreover, we define the $\mathbb{Z}$-wta $\mathcal{A}=(Q, \Sigma, X, \delta, \kappa)$, where $Q=$ $\{p, q, r\}, \Sigma=\Sigma_{1}=\{\gamma\}, X=\{x\}$. Moreover,

- $\delta_{0}(x, p)=-1, \delta_{0}(x, q)=\delta_{0}(x, r)=1$,

- $\delta_{1}(p, \gamma, p)=\delta_{1}(q, \gamma, q)=1$ and $\delta_{1}(s, \gamma, t)=0$ for every other combination $s, t \in Q$,

- $\kappa(p)=\kappa(q)=\kappa(r)=1$.

There are three runs $\omega_{p}, \omega_{q}$, and $\omega_{r}$ on the input tree $x$, which are defined by $\omega_{p}(\varepsilon)=p, \omega_{q}(\varepsilon)=q$, and $\omega_{r}(\varepsilon)=r$. For these runs, we have

$$
\delta^{*}\left(\omega_{p}\right) \cdot \kappa(p)+\delta^{*}\left(\omega_{q}\right) \cdot \kappa(q)+\delta^{*}\left(\omega_{r}\right) \cdot \kappa(r)=(-1) \cdot 1+1 \cdot 1+1 \cdot 1=1,
$$

hence $\|\mathcal{A}\|(x)=1$. For each $n \geq 1$, there are two runs $\omega_{p, n}$ and $\omega_{q, n}$ with nonzero weight on the tree $\gamma^{n}(x)$. The run $\omega_{p, n}$ associates $p$ with each position in $\gamma^{n}(x)$, and the run $\omega_{q, n}$ is defined analogously. For these runs, we have

$$
\delta^{*}\left(\omega_{p, n}\right) \cdot \kappa(p)+\delta^{*}\left(\omega_{q, n}\right) \cdot \kappa(q)=(-1) \cdot 1+1 \cdot 1=0,
$$

hence $\|\mathcal{A}\|\left(\gamma^{n}(x)\right)=0$. This means that $T_{\|\mathcal{A}\|}(x)=\{x\}$ and $T_{\|\mathcal{A}\|}(w)=\emptyset$ for every $w \in X^{*}$ with $w \neq x$. Hence $\mathcal{A}$ is summable.

However, for every $\xi \in T_{\Sigma}(X)$, we have $\operatorname{yd}_{\Sigma}(\xi)=x$ and there is a run $\omega$ on $\xi$ such that $\delta^{*}(\omega) \cdot \kappa(\omega(\varepsilon)) \neq 0$. Hence the set $U_{\mathcal{A}}(x)$ is infinite and thus $\mathcal{A}$ is not plain.

Now we turn to local weighted tree languages and the weighted languages determined by them.

We call a $K$-weighted local system $\mathcal{L}=(\Sigma, X, \varphi, \rho)$ plain if $K$ is complete or the set $\left\{\xi \in \operatorname{yd}_{\Sigma}^{-1}(w) \mid \varphi(\xi) \cdot \rho(\operatorname{rt}(\xi)) \neq 0\right\}$ is finite for every $w \in X^{*}$. It follows immediately from the corresponding definitions that a $K$-wls $\mathcal{L}$ is plain if and only if $\|\mathcal{L}\|$ is summable. For a plain $K$-wls $\mathcal{L}$, we call $\operatorname{yd}(\|\mathcal{L}\|)$ the weighted language determined by $\mathcal{L}$ and denote it by $\lambda_{\mathcal{L}}$. We denote by $\operatorname{Loc}_{\mathrm{p}}(K)$ the class of weighted tree languages determined by plain $K$-weighted local systems.

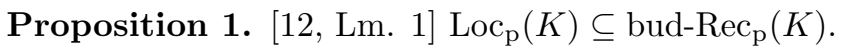


Proof. The construction used in the proof of [14, Thm. II. 9.4] (see also Lemma 1 of [12]) can be naturally extended to the yield alphabet. Indeed, let $\mathcal{L}=(\Sigma, X, \varphi, \rho)$ be a $K$-wls and construct the $K$-wta $\mathcal{A}=(Q, \Sigma, X, \delta, \kappa)$ in the following way. Let $Q=\{\bar{z} \mid z \in(\Sigma \cup X)\}$ and, for every $y \in\left(\Sigma_{0} \cup X\right)$ and $z \in(\Sigma \cup X)$, let $\delta_{0}(y, \bar{z})=\varphi_{0}(y)$, if $z=y$ and let $\delta_{0}(y, \bar{z})=0$, otherwise. Furthermore, for every $k \geq 1, z_{1} \ldots z_{k} \in(\Sigma \cup X)^{k}, \sigma \in \Sigma_{k}$, and $z \in(\Sigma \cup X)$, let

$$
\delta_{k}\left(\overline{z_{1}} \ldots \overline{z_{k}}, \sigma, \bar{z}\right)= \begin{cases}\varphi_{k}\left(z_{1} \ldots z_{k}, \sigma\right) & \text { if } z=\sigma \\ 0 & \text { otherwise }\end{cases}
$$

Lastly, for every $\sigma \in \Sigma$, let $\kappa(\bar{\sigma})=\rho(\sigma)$.

It is easy to see that $\mathcal{A}$ is bu-deterministic. Now let $\xi \in T_{\Sigma}(X)$ and $\omega_{\xi} \in R_{\mathcal{A}}(\xi)$ be the run defined by $\omega_{\xi}(p)=\overline{\xi(p)}$, for every $p \in \operatorname{pos}(\xi)$. It can be readily seen by induction on $\xi$ that $\delta^{*}\left(\omega_{\xi}\right)=\varphi(\xi)$. Moreover, for every run $\omega \in R_{\mathcal{A}}(\xi)$ with $\omega \neq \omega_{\xi}$, we have $\delta^{*}(\omega)=0$. Then, for every $\xi$ in $T_{\Sigma}(X)$, we get that

$$
\begin{aligned}
&\|\mathcal{A}\|(\xi)=\sum_{\omega \in \mathcal{R}_{\mathcal{A}}(\xi)} \delta^{*}(\omega) \cdot \kappa(\omega(\varepsilon))= \delta^{*}\left(\omega_{\xi}\right) \cdot \kappa\left(\omega_{\xi}(\varepsilon)\right)= \\
& \delta^{*}\left(\omega_{\xi}\right) \cdot \kappa(\overline{\xi(\varepsilon)})=\varphi(\xi) \cdot \rho(\operatorname{rt}(\xi))=\|\mathcal{L}\|(\xi) .
\end{aligned}
$$

Now assume that $\mathcal{L}$ is plain. By our above remark, $\|\mathcal{L}\|$ is summable. Hence $\|\mathcal{A}\|$ is also summable. Since $\mathcal{A}$ is bu-deterministic, by Lemma $1(3)$ we obtain that $\mathcal{A}$ is plain.

Now we have all the concepts available to state the main result of this paper.

Theorem 1. For each weighted language $\lambda: T_{\Sigma}(X) \rightarrow K$, the following five statements are equivalent:

(1) $\lambda$ can be generated by a plain $\mathrm{CF}(K)$-grammar,

(2) $\lambda$ can be determined by a plain $K$-wls,

(3) $\lambda$ can be recognized by a plain and bottom-up deterministic $K$-wta,

(4) $\lambda$ can be recognized by a plain and top-down deterministic $K$-wta,

(5) $\lambda$ can be recognized by a plain $K$-wta.

If $K$ is zero-sum free, then the following can be added to the list:

(6) $\lambda$ can be recognized by a $K$-wta $\mathcal{A}$ such that $\|\mathcal{A}\|$ is summable.

Proof. The proof of the first statement is that $(1) \Rightarrow(2)$ by Lemma $3,(2) \Rightarrow(3)$ by Proposition $1,(1) \Rightarrow(4)$ by Lemma $4,(3),(4) \Rightarrow(5)$ by definition, and finally $(5) \Rightarrow(1)$ by Lemma 6 . The second statement follows from Corollary 1(2).

Lemma 3. $\mathrm{CFL}_{\mathrm{p}}(K) \subseteq \operatorname{yd}\left(\operatorname{Loc}_{\mathrm{p}}(K)\right)$. 
Proof. Let $\mathcal{G}=(N, X, Z, P$, wt $)$ be a plain $\mathrm{CF}(K)$-grammar. We define the $K$-wls $\mathcal{L}=(\bar{P}, X, \varphi, \rho)$, where

- $\bar{P}$ is the ranked alphabet defined in Section 2.2,

- for every $k \geq 1$, the mapping $\varphi_{k}: \operatorname{Fork}_{k}(\bar{P} \cup X) \rightarrow K$ is defined by $\varphi_{k}\left(y_{1} \ldots y_{k}, r\right)=\operatorname{wt}(r)$ if $r=\left(A \rightarrow \alpha_{1} \ldots \alpha_{k}\right)$ for some $k \geq 1$ and $\alpha_{1}, \ldots, \alpha_{n} \in(N \cup X)$, and

$$
y_{i}= \begin{cases}\alpha_{i} & \text { if } \alpha_{i} \in X \\ \text { a rule } r_{i} \in P \text { with } \operatorname{lhs}\left(r_{i}\right)=\alpha_{i} & \text { if } \alpha_{i} \in N\end{cases}
$$

for every $1 \leq i \leq k$; and $\varphi_{k}\left(y_{1} \ldots y_{k}, r\right)=0$ in every other case,

- the mapping $\varphi_{0}: \operatorname{Fork}_{0}(\bar{P} \cup X) \rightarrow K$ is defined by $\varphi_{0}(r)=\operatorname{wt}(r)$ for every $r \in \bar{P}_{0}$ and $\varphi_{0}(x)=1$ for every $x \in X$,

- the root mapping $\rho:(\bar{P} \cup X) \rightarrow K$ is defined, for every $y \in(\bar{P} \cup X)$ by $\rho(y)=1$ if $y \in P$ with $\operatorname{lhs}(y)=Z$ and $\rho(y)=0$ in every other case.

Let $w \in X^{*}$. Due to the construction, $D_{\mathcal{G}}(w) \subseteq \operatorname{yd}_{\bar{P}}^{-1}(w)$ and

$$
\varphi(\zeta) \cdot \rho(\operatorname{rt}(\zeta))= \begin{cases}\mathrm{wt}(\zeta) & \text { if } \zeta \in D_{\mathcal{G}}(w) \\ 0 & \text { otherwise }\end{cases}
$$

for every $\zeta \in \operatorname{yd}_{\bar{P}}^{-1}(w)$. If $K$ is not complete, then the set $\left\{\zeta \in D_{\mathcal{G}}(w) \mid \operatorname{wt}(\zeta) \neq 0\right\}$ is finite because $\mathcal{G}$ is plain. Thus the set $\left\{\zeta \in \operatorname{yd}_{\Sigma}^{-1}(w) \mid \varphi(\zeta) \cdot \rho(\operatorname{rt}(\zeta)) \neq 0\right\}$ is also finite. Hence $\mathcal{L}$ is plain. Moreover, we have

$$
\begin{gathered}
\lambda_{\mathcal{G}}(w)=\sum_{\zeta \in D_{\mathcal{G}}(w), \operatorname{wt}(\zeta) \neq 0} \operatorname{wt}(\zeta)=\sum_{\zeta \in \operatorname{yd}_{\bar{P}}^{-1}(w)} \varphi(\zeta) \cdot \rho(\operatorname{rt}(\zeta))= \\
\sum_{\zeta \in \operatorname{yd}_{\bar{P}}^{-1}(w)}\|\mathcal{L}\|(\zeta)=\operatorname{yd}(\|\mathcal{L}\|)(w),
\end{gathered}
$$

where second equality follows using that $D_{\mathcal{G}}(w) \subseteq \operatorname{yd}_{\bar{P}}^{-1}(w)$ and the note made on the values of $\varphi(\zeta) \cdot \rho(\operatorname{rt}(\zeta))$ for trees not in $D_{\mathcal{G}}(w)$.

Lemma 4. $\operatorname{CFL}_{\mathrm{p}}(K) \subseteq \operatorname{yd}\left(\operatorname{bud}_{-R_{e c}}(K) \cap \operatorname{tdd}-\operatorname{Rec}_{\mathrm{p}}(K)\right)$.

Proof. Let $\mathcal{G}=(N, X, Z, P$, wt $)$ be a plain $\mathrm{CF}(K)$-grammar. We define the $K$-wta $\mathcal{A}=(Q, \bar{P}, X, \delta, \kappa)$, where

- $Q=N \cup\{\bar{x} \mid x \in X\}$,

- $\bar{P}$ is the ranked alphabet defined in Section 2.2,

- the family $\delta$ is defined as follows: 
- for every $k \geq 1$, rule $r=\left(A \rightarrow \alpha_{1} \ldots \alpha_{k}\right) \in \bar{P}_{k}$ with $\alpha_{1}, \ldots, \alpha_{k} \in$ $(N \cup X)$, and $q_{1}, \ldots, q_{k}, q \in Q$, we let $\delta_{k}\left(q_{1} \ldots q_{k}, r, q\right)=\operatorname{wt}(r)$ if $q=A$ and

$$
q_{i}= \begin{cases}\alpha_{i} & \text { if } \alpha_{i} \in N \\ \bar{x} & \text { if } \alpha_{i}=x \in X,\end{cases}
$$

for $1 \leq i \leq k$; and we let $\delta_{k}\left(q_{1} \ldots q_{k}, r, q\right)=0$ for every other choice of $q_{1}, \ldots, q_{k}$ and $q$,

- for every $r=(A \rightarrow \varepsilon) \in \bar{P}_{0}$ and $q \in Q$, we define $\delta_{0}(r, q)=\operatorname{wt}(r)$ if $q=A$ and $\delta_{0}(r, q)=0$ otherwise,

- for every $x \in X$ and $q \in Q$, we define $\delta_{0}(x, q)=1$ if $q=\bar{x}$ and $\delta_{0}(x, q)=$ 0 otherwise,

- for every $q \in Q, \kappa(q)=1$ if $q=Z$ and $\kappa(q)=0$ otherwise.

It is obvious that $\mathcal{A}$ is both bu-deterministic and td-deterministic. We will show that it is also plain and that $\lambda_{\mathcal{G}}=\operatorname{yd}(\|\mathcal{A}\|)$.

For every $\zeta \in T_{\bar{P}}(X)$, there is a distinguished run $\omega_{\zeta} \in R_{\mathcal{A}}(\zeta)$ defined for each $p \in \operatorname{pos}(\zeta)$ by

$$
\omega_{\zeta}(p)= \begin{cases}\operatorname{lhs}(r) & \text { if } \zeta(p)=r \text { for some } r \in \bar{P} \\ \bar{x} & \text { if } \zeta(p)=x \text { for some } x \in X .\end{cases}
$$

The transition mappings of $\mathcal{A}$ are designed in such a way that, for every $\zeta \in T_{\bar{P}}(X)$ and $\omega \in R_{\mathcal{A}}(\zeta)$, we have $\delta^{*}(\omega)=0$ if $\omega \neq \omega_{\zeta}$, and

$$
\delta^{*}\left(\omega_{\zeta}\right) \cdot \kappa\left(\omega_{\zeta}(\varepsilon)\right)= \begin{cases}\operatorname{wt}(\zeta) & \text { if } \zeta \in D_{\mathcal{G}}(w) \text { for some } w \in X^{*} \\ 0 & \text { otherwise. }\end{cases}
$$

This, the fact that $\mathcal{G}$ is plain, and that $D_{\mathcal{G}}(w) \subseteq \operatorname{yd}_{\bar{P}}^{-1}(w)$ implies that if $K$ is not complete, then the set $\left\{\zeta \in \operatorname{yd}_{\bar{P}}^{-1}(w) \mid \delta^{*}\left(\omega_{\zeta}\right) \cdot \kappa\left(\omega_{\zeta}(\varepsilon)\right) \neq 0\right\}$ is finite for every $w \in X^{*}$. This means that $\mathcal{A}$ is plain. Furthermore, for every $w \in X^{*}$, we have

$$
\begin{aligned}
\lambda_{\mathcal{G}}(w)= & \sum_{\zeta \in D_{\mathcal{G}}(w), \operatorname{wt}(\zeta) \neq 0} \operatorname{wt}(\zeta)=\sum_{\zeta \in \operatorname{yd}_{\bar{P}}^{-1}(w)} \delta^{*}\left(\omega_{\zeta}\right) \cdot \kappa\left(\omega_{\zeta}(\varepsilon)\right)= \\
& \sum_{\zeta \in \operatorname{yd}_{\bar{P}}^{-1}(w)} \sum_{\omega \in R_{\mathcal{A}}(\zeta)} \delta^{*}(\omega) \cdot \kappa(\omega(\varepsilon))=\sum_{\zeta \in \operatorname{yd}_{\bar{P}}^{-1}(w)}\|\mathcal{A}\|(\zeta)=\operatorname{yd}(\|\mathcal{A}\|)(w),
\end{aligned}
$$

where $\omega_{\zeta}$ is the particular run in $R_{\mathcal{A}}(\zeta)$ defined above. The second equality holds because $D_{\mathcal{G}}(w) \subseteq \operatorname{yd}_{\bar{P}}^{-1}(w)$ and the note made on $\delta^{*}\left(\omega_{\zeta}\right)$. The third one holds because $\delta^{*}(\omega)=0$ for $\omega \neq \omega_{\zeta}$.

To prove that $\mathrm{yd}\left(\operatorname{Rec}_{\mathrm{p}}(K)\right) \subseteq \mathrm{CFL}_{\mathrm{p}}(K)$ we need the following preparation. A $K$-wta $\mathcal{A}=(Q, \Sigma, X, \delta, \kappa)$ has Boolean root weights (see [13, Sec. 3.2]) if $\kappa(q) \in$ $\{0,1\}$ for every $q \in Q$. In this case we replace $\kappa$ by the set $F=\{q \in Q \mid \kappa(q)=1\}$ 
and write $\mathcal{A}=(Q, \Sigma, X, \delta, F)$. For a $\xi \in T_{\Sigma}(X)$, let $R_{\mathcal{A}}^{F}(\xi)=\left\{\omega \in R_{\mathcal{A}}(\xi) \mid \omega(\varepsilon) \in\right.$ $F\}$. Then it is easy to see that $\|\mathcal{A}\|(\xi)=\sum_{\omega \in R_{\mathcal{A}}^{F}(\xi)} \delta^{*}(\omega)$. Moreover,

$$
U_{\mathcal{A}}(w)=\left\{\xi \in \operatorname{yd}_{\Sigma}^{-1}(w) \mid \exists\left(\omega \in R_{\mathcal{A}}^{F}(\xi)\right): \delta^{*}(\omega) \neq 0\right\}
$$

and

$$
\lambda_{\mathcal{A}}(w)=\sum_{\xi \in U_{\mathcal{A}}(w), \omega \in R_{\mathcal{A}}^{F}(\xi)} \delta^{*}(\omega)
$$

In $[4$, Thm. 6.1.6] it is shown that $K$-wta and $K$-wta with Boolean root weights are equally powerful (see [13, Thm. 3.6]). We will now give another, slightly modified proof.

Lemma 5. For each $K$-wta $\mathcal{A}$ there is a $K$-wta $\mathcal{A}^{\prime}$ with Boolean root weights such that $\|\mathcal{A}\|=\left\|\mathcal{A}^{\prime}\right\|$ and $U_{\mathcal{A}}(w)=U_{\mathcal{A}^{\prime}}(w)$ for every $w \in \Sigma^{*}$.

Proof. Let $\mathcal{A}=(Q, \Sigma, X, \delta, \kappa)$ be a $K$-wta. We construct a $K$-wta $\mathcal{A}^{\prime}$ with Boolean root weights such that $\|\mathcal{A}\|=\left\|\mathcal{A}^{\prime}\right\|$. First, let $F=\left\{q_{f} \mid q \in Q\right\}$ be a disjoint copy of $Q$ and let $Q^{\prime}=Q \cup F$. Then construct $\mathcal{A}^{\prime}=\left(Q^{\prime}, \Sigma, X, \delta^{\prime}, F\right)$, where $\delta^{\prime}$ is defined as follows:

- for every $y \in\left(\Sigma_{0} \cup X\right)$ and $q \in Q$, let

$$
\delta_{0}^{\prime}(y, q)=\delta_{0}(y, q) \text { and } \delta_{0}^{\prime}\left(y, q_{f}\right)=\delta_{0}(y, q) \cdot \kappa(q), \text { and }
$$

- for every $k \geq 1, \sigma \in \Sigma_{k}, q_{1}, \ldots, q_{k} \in Q^{\prime}$, and $q \in Q$, let

$$
\delta_{k}^{\prime}\left(q_{1} \ldots q_{k}, \sigma, q\right)= \begin{cases}\delta_{k}\left(q_{1} \ldots q_{k}, \sigma, q\right) & \text { if } q_{1}, \ldots, q_{k} \in Q \\ 0 & \text { otherwise }\end{cases}
$$

and

$$
\delta_{k}^{\prime}\left(q_{1} \ldots q_{k}, \sigma, q_{f}\right)= \begin{cases}\delta_{k}\left(q_{1} \ldots q_{k}, \sigma, q\right) \cdot \kappa(q) & \text { if } q_{1}, \ldots, q_{k} \in Q \\ 0 & \text { otherwise. }\end{cases}
$$

Now we will explore the relation between the runs of $\mathcal{A}$ and of $\mathcal{A}^{\prime}$ on a tree $\xi \in$ $T_{\Sigma}(X)$. First, we note that $R_{\mathcal{A}}(\xi) \subseteq R_{\mathcal{A}^{\prime}}(\xi)$ because $Q \subseteq Q^{\prime}$. Actually, a run $\omega \in R_{\mathcal{A}^{\prime}}(\xi)$ is in $R_{\mathcal{A}}(\xi)$ if and only if $\omega(p) \in Q$ for every $p \in \operatorname{pos}(\xi)$. Next, we introduce the notation

$$
\widehat{R}_{\mathcal{A}^{\prime}}^{F}(\xi)=\left\{\omega \in R_{\mathcal{A}^{\prime}}^{F}(\xi) \mid \omega(p) \in Q \text { for every } p \in \operatorname{pos}(\xi) \text { with } p \neq \varepsilon\right\} .
$$

Note that, for each $\omega \in \widehat{R}_{\mathcal{A}^{\prime}}^{F}(\xi)$, we have $\omega(\varepsilon)=q_{f}$ for some $q \in Q$. Moreover, there is a bijection from $\widehat{R}_{\mathcal{A}^{\prime}}^{F}(\xi)$ to $R_{\mathcal{A}}(\xi)$ defined by the correspondence $\omega \mapsto \widehat{\omega}$, where $\widehat{\omega}(\varepsilon)=q$ if $\omega(\varepsilon)=q_{f}$ and $\widehat{\omega}(p)=\omega(p)$ for any other $p \in \operatorname{pos}(\xi)$ with $p \neq \varepsilon$. 
It follows from the construction that, for every $\xi \in T_{\Sigma}(X)$ and $\omega \in R_{\mathcal{A}^{\prime}}(\xi)$, we have

$$
\delta^{\prime *}(\omega)=\left\{\begin{array}{lc}
\delta^{*}(\omega) & \text { if } \omega \in R_{\mathcal{A}}(\xi) \\
\delta^{*}(\widehat{\omega}) \cdot \kappa(\widehat{\omega}(\varepsilon)) & \text { if } \omega \in \widehat{R}_{\mathcal{A}^{\prime}}^{F}(\xi) \\
0 & \text { otherwise }
\end{array}\right.
$$

where $\omega \mapsto \widehat{\omega}$ is the bijection defined above.

Then we find that

$$
\left\|\mathcal{A}^{\prime}\right\|(\xi)=\sum_{\omega \in R_{\mathcal{A}^{\prime}}^{F}(\xi)} \delta^{\prime *}(\omega)=\sum_{\omega \in \widehat{R}_{\mathcal{A}^{\prime}}^{F}(\xi)} \delta^{\prime *}(\omega)=\sum_{\omega \in R_{\mathcal{A}}(\xi)} \delta^{*}(\omega) \cdot \kappa(\omega(\varepsilon))=\|\mathcal{A}\|(\xi)
$$

holds for every $\xi \in T_{\Sigma}(X)$. The second equality holds because $\delta^{*}(\omega)=0$ for each $\omega \in\left(R_{\mathcal{A}^{\prime}}^{F}(\xi) \backslash \widehat{R}_{\mathcal{A}^{\prime}}^{F}(\xi)\right)$ and the third one holds by the bijection between $\widehat{R}_{\mathcal{A}^{\prime}}^{F}(\xi)$ and $R_{\mathcal{A}}(\xi)$ described above. This proves that $\left\|\mathcal{A}^{\prime}\right\|=\|\mathcal{A}\|$.

Now, let $w \in X^{*}$. To see that $U_{\mathcal{A}}(w)=U_{\mathcal{A}^{\prime}}(w)$, first we note that

$$
U_{\mathcal{A}^{\prime}}(w)=\left\{\xi \in \operatorname{yd}_{\Sigma}^{-1}(w) \mid \exists\left(\omega \in \widehat{R}_{\mathcal{A}^{\prime}}^{F}(\xi)\right): \delta^{\prime *}(\omega) \neq 0\right\}
$$

Due to the bijection between $\widehat{R}_{\mathcal{A}^{\prime}}^{F}(\xi)$ and $R_{\mathcal{A}}(\xi)$ for every $\xi \in T_{\Sigma}(X)$, we have $U_{\mathcal{A}^{\prime}}(w)=U_{\mathcal{A}}(w)$.

Corollary 2. For each plain $K$-wta $\mathcal{A}$ there is a plain $K$-wta $\mathcal{A}^{\prime}$ with Boolean root weights such that $\lambda_{\mathcal{A}}=\lambda_{\mathcal{A}^{\prime}}$.

Proof. Let $\mathcal{A}$ be a plain $K$-wta and construct $\mathcal{A}^{\prime}$ as in Lemma 5. Since $U_{\mathcal{A}}(w)=$ $U_{\mathcal{A}^{\prime}}(w)$ for every $w \in \Sigma^{*}$, it follows that $\mathcal{A}^{\prime}$ is also plain. Furthermore, since $\|\mathcal{A}\|=\|\mathcal{A}\|^{\prime}$, we have

$$
\lambda_{\mathcal{A}}=\operatorname{yd}(\|\mathcal{A}\|)=\operatorname{yd}\left(\left\|\mathcal{A}^{\prime}\right\|\right)=\lambda_{\mathcal{A}^{\prime}} .
$$

Lemma 6. $\operatorname{yd}\left(\operatorname{Rec}_{\mathrm{p}}(K)\right) \subseteq \operatorname{CFL}_{\mathrm{p}}(K)$.

Proof. Let $\mathcal{A}=(Q, \Sigma, X, \delta, F)$ be a plain $K$-wta with Boolean root weights (by Corollary 2 without loss of generality). We construct a plain $\mathrm{CF}(K)$-grammar $\mathcal{G}$ such that $\lambda_{\mathcal{A}}=\lambda_{\mathcal{G}}$. Let $\mathcal{G}=(N, X, Z, P$, wt $)$, where

- $N=\{Z\} \cup(Q \times(\Sigma \cup X))$, where $Z$ is a new symbol,

- $P$ and wt are defined as follows:

- for every $(q, y) \in F \times(\Sigma \cup X)$, the rule $r=(Z \rightarrow(q, y))$ is in $P$ with $\operatorname{wt}(r)=1$,

- for every $k \geq 1,(q, \sigma) \in Q \times \Sigma_{k},\left(q_{1}, y_{1}\right), \ldots,\left(q_{k}, y_{k}\right) \in Q \times(\Sigma \cup X)$, the rule $r=\left((q, \sigma) \rightarrow\left(q_{1}, y_{1}\right) \ldots\left(q_{k}, y_{k}\right)\right)$ is in $P$ with $\operatorname{wt}(r)=$ $\delta_{k}\left(q_{1} \ldots q_{k}, \sigma, q\right)$, 


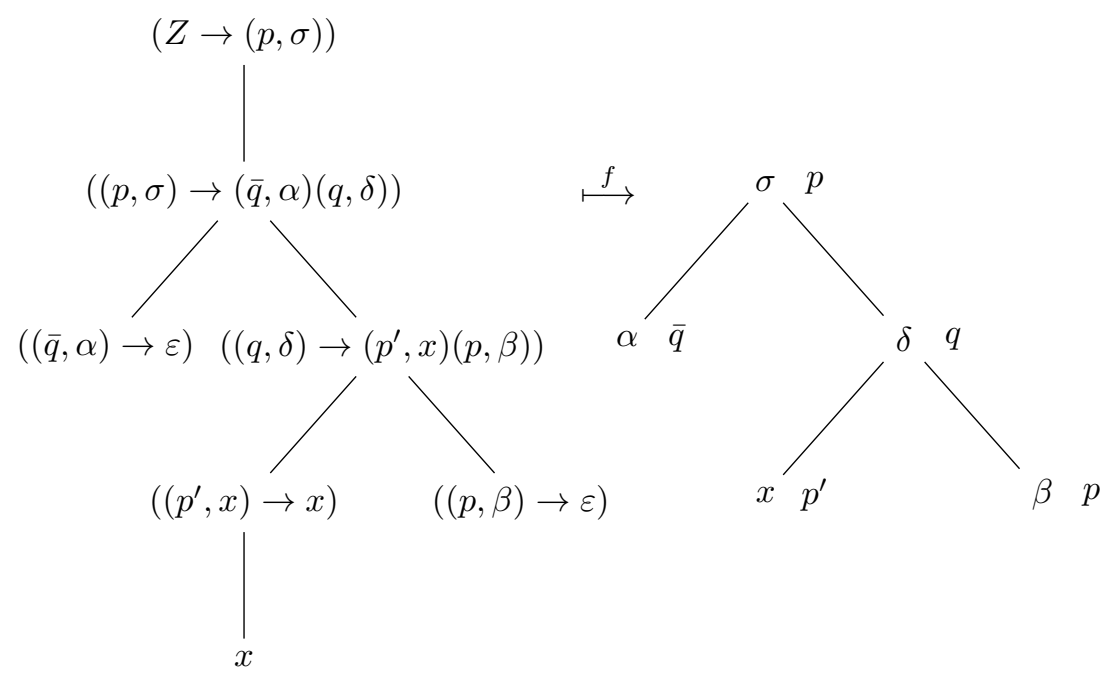

Figure 2: A visualization of the bijection $f$ given in Lemma 6 .

- for every $(q, \sigma) \in Q \times \Sigma_{0}$, the rule $r=((q, \sigma) \rightarrow \varepsilon)$ is in $P$ with wt $(r)=$ $\delta_{0}(\sigma, q)$, and

- for every $(q, x) \in Q \times X$, the rule $r=((q, x) \rightarrow x)$ is in $P$ with wt $(r)=$ $\delta_{0}(x, q)$.

First we show that, for every $w \in X^{*}$, there is a bijection $f$ between the sets

$$
D_{\mathcal{G}}(w) \text { and }\left\{(\xi, \omega) \mid \xi \in \operatorname{yd}_{\Sigma}^{-1}(w), \omega \in R_{\mathcal{A}}^{F}(\xi)\right\}
$$

such that if $f(\zeta)=(\xi, \omega)$ for $\zeta \in D_{\mathcal{G}}(w)$, then $\operatorname{wt}(\zeta)=\delta^{*}(\omega)$. To find such a bijection, for each tree $\zeta \in D_{\mathcal{G}}(w)$, we define $\hat{\zeta} \in T_{\Sigma}(X)$ and $\omega_{\zeta} \in R_{\mathcal{A}}^{F}(\hat{\zeta})$ as follows. Let $\operatorname{pos}(\hat{\zeta})=\left\{p \in \operatorname{pos}\left(\left.\zeta\right|_{1}\right) \mid\left(\left.\zeta\right|_{1}\right)(p) \notin X\right\}$. Moreover, for every $p \in \operatorname{pos}(\hat{\zeta})$, let $\hat{\zeta}(p)$ be the second, while $\omega_{\zeta}(p)$ be the first component of $\operatorname{lhs}(r)$, where $r=\left(\left.\zeta\right|_{1}\right)(p)$ (see Figure 2 for example). It can be seen that the mapping $f: \zeta \mapsto\left(\hat{\zeta}, \omega_{\zeta}\right)$ is a bijection which satisfies the condition $\mathrm{wt}(\zeta)=\delta^{*}\left(\omega_{\zeta}\right)$.

Now, assume that $K$ is not complete and let $w \in X^{*}$. Since $\mathcal{A}$ is plain and thus $U_{\mathcal{A}}(w)$ is finite, the set $\left\{(\xi, \omega) \mid \xi \in \operatorname{yd}_{\Sigma}^{-1}(w), \omega \in R_{\mathcal{A}}^{F}(\xi), \delta^{*}(\omega) \neq 0\right\}$ is also finite because, for every $\xi \in \operatorname{yd}_{\Sigma}^{-1}(w)$, the set $R_{A}^{F}(\xi)$ is also finite. Then, due to the bijection defined above, the set $\left\{\zeta \in D_{\mathcal{G}}(w) \mid \operatorname{wt}(\zeta) \neq 0\right\}$ is also finite, which proves that $\mathcal{G}$ is plain. 
Finally, we show that $\lambda_{\mathcal{G}}=\lambda_{\mathcal{A}}$. Indeed, for every $w \in X^{*}$,

$$
\begin{gathered}
\lambda_{\mathcal{G}}(w)=\sum_{\zeta \in D_{\mathcal{G}}(w), \operatorname{wt}(\zeta) \neq 0} \operatorname{wt}(\zeta)=\sum_{\substack{\xi \in \mathrm{yd}_{\Sigma}^{-1}(w) \\
\omega \in R_{\mathcal{A}}^{F}(\xi), \delta^{*}(\omega) \neq 0}} \delta^{*}(\omega)= \\
\sum_{\xi \in U_{\mathcal{A}}(w), \omega \in R_{\mathcal{A}}^{F}(\xi)} \delta^{*}(\omega)=\lambda_{\mathcal{A}}(w),
\end{gathered}
$$

where the second equality holds due to the bijection $f$ defined above, the third one holds because we extend the sum with finitely many 0 , and the fourth one holds from the definition of $\lambda_{\mathcal{A}}$ and Lemma 1(1).

\section{References}

[1] A. Alexandrakis and S. Bozapalidis, Weighted grammars and Kleene's theorem. Information Processing Letters, 24(1):1-4, January 1987.

[2] J. Berstel and C. Reutenauer, Recognizable formal power series on trees. Theoret. Comput. Sci., 18(2):115-148, 1982.

[3] B. Borchardt, A Pumping Lemma and Decidability Problems for Recognizable Tree Series. Acta Cyb., 16(4):509-544, 2004.

[4] B. Borchardt, The Theory of recognizable Tree Series. Verlag für Wissenschaft und Forschung, Berlin, 2005 (PhD thesis, TU Dresden, Germany, 2004).

[5] N. Chomsky and M.P. Schützenberger, The algebraic theory of context-free languages. In: Computer Programming and Formal Systems (eds. P. Braffort and D. Hirschberg), North-Holland, 118-161, North-Holland, 1963.

[6] H. Comon, M. Dauchet, R. Gilleron, F. Jacquemard, D. Lugiez, S. Tison, and M. Tommasi, Tree Automata Techniques and Applications. Available on: http://www.grappa.univ-lille3.fr/tata, 1997.

[7] J. Doner, Tree acceptors and some of their applications. J. Comput. System Sci., 4 (1970) 406-451.

[8] M. Droste and H. Vogler, The Chomsky-Schützenberger theorem for quantitative context-free languages. Int. J. of Foundations of Comput. Sci., 25(8):955969, 2014.

[9] S. Eilenberg, Automata, Languages, and Machines - Volume A. Academic Press, 1974, Pure and Applied Mathematics, 59.

[10] J. Engelfriet, Context-free grammars with storage. Technical Report 86-11, University of Leiden, 1986. See also: arXiv:1408.0683 [cs.FL], 2014. 
[11] Z. Ésik and W. Kuich, Formal tree series. J. of Automata, Languages, and Combinatorics, 8(2):219-285, 2003.

[12] Z. Fülöp, Local Weighted Tree Languages. Acta Cybernetica, 22 (2015) 393402.

[13] Z. Fülöp and H. Vogler, Weighted tree automata and tree transducers. in: Handbook of Weighted Automata (eds. M. Droste, W. Kuich, and H. Vogler), chapter 9, 313-403, Springer-Verlag, 2009.

[14] F. Gécseg and M. Steinby, Tree Automata. Akadémiai Kiadó, 1984. See also: arXiv:1509.06233v1 [cs.FL] 21 Sep 2015.

[15] F. Gécseg and M. Steinby, Tree languages. in: Handbook of Formal Languages (eds. G. Rozenberg and A. Salomaa), Vol. 1, pp.1-68, Springer-Verlag, 1997.

[16] J.A. Goguen, J.W. Thatcher, E.G. Wagner, and J.B. Wright, Initial algebra semantics and continuous algebras. J. ACM, 24:68-95, 1977.

[17] J.S. Golan, Semirings and their Applications. Kluwer Academic Publishers, Dordrecht, 1999.

[18] U. Hebisch and H.J. Weinert, Semirings - Algebraic Theory and Applications in Computer Science. World Scientific, Singapore, 1998.

[19] W. Kuich and A. Salomaa, Semirings, Automata, Languages. Monogr. Theoret. Comput. Sci. EATCS Series, Volume 5, Springer-Verlag, 1986.

[20] M. Magidor and G. Moran, Finite Automata over Finite Trees. Technical report 30, Hebrew University, Jerusalem, 1969.

[21] I. Petre and A. Salomaa, Algebraic systems and pushdown automata. In: Handbook of Weighted Automata (eds. M. Droste, W. Kuich, and H. Vogler), chapter 7, 257-289, Springer-Verlag, 2009.

[22] W.C. Rounds, Mappings and grammars on trees. Math. Systems Theory 4 (1970) 257-287.

[23] W.C. Rounds, Tree-oriented proofs of some theorems on context-free and indexed languages. 2nd Ann. Assoc. Comput. Sci. Symp. on Theory of Computing, 109-116, 1970.

[24] A. Salomaa and M. Soittola, Automata-Theoretic Aspects of Formal Power Series. Texts and Monographs in Computer Science, Springer-Verlag, 1978.

[25] M. Steinby, Algebras as tree automata. Record Coll. Universal Algebra, Szeged 1975, North-Holland, 441-455, 1977.

[26] J.W. Thatcher, Characterizing derivation trees of context-free grammars through a generalization of finite automata theory. J. Comput. Syst. Sci., 1 (1967) 317-322. 
[27] J.W. Thatcher, Generalized ${ }^{2}$ sequential machine maps. J. Comput. Syst. Sci., 4 (1970) 339-367.

[28] J.W. Thatcher, Tree automata: an informal survey. in Currents in the Theory of Computing (ed. A. V. Aho), 143-172, Prentice Hall, 1973.

Received 24th May 2018 